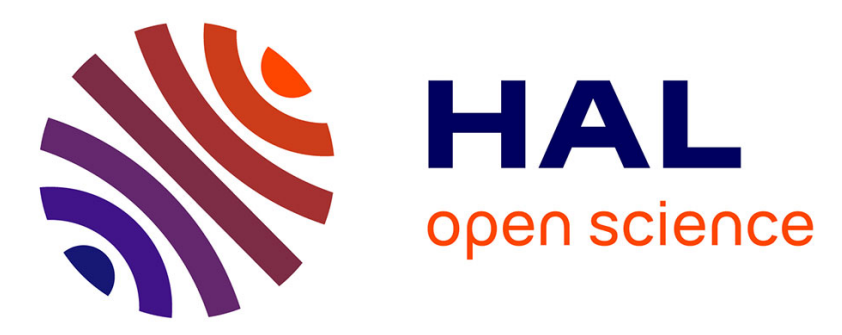

\title{
Mechanical identification of hyperelastic anisotropic properties of mouse carotid arteries
}

\author{
Pierre Badel, Stéphane Avril, Susan Lessner, Michael A. Sutton
}

\section{To cite this version:}

Pierre Badel, Stéphane Avril, Susan Lessner, Michael A. Sutton. Mechanical identification of hyperelastic anisotropic properties of mouse carotid arteries. 11th International Congress \& Exposition on Experimental and Applied Mechanics, 2011, Uncasville, United States. pp.7. hal-01063755

\section{HAL Id: hal-01063755 \\ https://hal.science/hal-01063755}

Submitted on 12 Sep 2014

HAL is a multi-disciplinary open access archive for the deposit and dissemination of scientific research documents, whether they are published or not. The documents may come from teaching and research institutions in France or abroad, or from public or private research centers.
L'archive ouverte pluridisciplinaire HAL, est destinée au dépôt et à la diffusion de documents scientifiques de niveau recherche, publiés ou non, émanant des établissements d'enseignement et de recherche français ou étrangers, des laboratoires publics ou privés. 


\title{
Mechanical identification of hyperelastic anisotropic properties of mouse carotid arteries
}

\author{
Badel Pierre $^{(\mathrm{a})}, \mathrm{PhD}$, Avril Stéphane ${ }^{(\mathrm{a})}, \mathrm{Pr}_{\text {, Lessner Susan }}^{(\mathrm{b})}, \mathrm{PhD}$, Sutton Michael $^{(\mathrm{c})}, \mathrm{Pr}$ \\ (a) Center for Health Engineering - Ecole des Mines de Saint Etienne - 158, cours Fauriel - 42023 Saint \\ Etienne - France \\ (b) Department of Cell Biology and Anatomy - University of South Carolina School of Medicine - \\ Columbia, South Carolina 29208, USA \\ ${ }^{(c)}$ Department of Mechanical Engineering - University of South Carolina - Columbia, South Carolina \\ 29208, USA
}

\begin{abstract}
The role of mechanics is known to be of primary order in many arterial diseases; however determining mechanical properties of arteries remains a challenge. This paper discusses the identifiability of a Holzapfel-type material model for a mouse carotid artery, using an inverse method based on a finite element model and 3D digital image correlation measurements of the surface strain during an inflation/extension test. Layer-specific mean fiber angles are successfully determined using a five parameter constitutive model, demonstrating good robustness of the identification procedure. Importantly, we show that a model based on a single thick layer is unable to render the biaxial mechanical response of the artery tested here. On the contrary, difficulties related to the identification of a seven parameter constitutive model are evidenced; such a model leads to multiple solutions. Nevertheless, it is shown that an additional mechanical test, different in nature with the previous one, solves this problem.
\end{abstract}

\section{Introduction}

Identification of mechanical and structural properties of the arteries is a major topic in cardiovascular research. Many arterial disorders involve significant changes in vascular mechanical properties. Not only do the structure and mechanical response of arteries vary according to many factors such as the location in the vasculature and age, but also their properties may alter under various physiological conditions and during the development of diseases [1,2]. Accurate mechanical identification of the arteries can therefore provide helpful information for clinical diagnoses and treatments. To improve the contribution of solid mechanics, a lot of effort has been undertaken to develop constitutive models of the arterial wall as well as experimental and numerical methods to identify these models. 
Several constitutive models intended to describe the mechanical response of arterial tissues at finite strains have been developed, see [3] for an extensive review of these models. Most of anisotropic non-linear models considering the passive response of arteries are hyperelastic. Fung [1] first introduced a phenomenological exponential strain energy function. More recently, structurally-motivated models including fiber reinforcements have been developed. Bischoff [4] suggested representing the nonlinear orthotropic material response of the arterial wall, with a homogeneous orthotropic model. This model has proven adequate to capture the nonlinear orthotropic response of vascular tissues although the physical meaning of its parameters is not clear [5]. Holzapfel [6] introduced a two-fiber family model to account for the helically-oriented distribution of collagen fibers within the arterial wall. To ideally describe the arterial wall from the mechanical point of view, two separate layers of this material are required for medial and adventitial layers.

Originally, Holzapfel [6] grounded the choice of two symmetrically- and helically-oriented fiber families per layer on histological observations. Since the compositions of medial and adventitial layers (elastin, collagen and cell contents) are different [2], two separate layers must be distinguished. Note also that some authors introduced the active response of the arterial wall due to smooth muscle activity [7], which is not the concern of this paper.

Correct identification of the parameters of the constitutive model is a key issue in considering the reliability of interpretation for medical purposes or subsequent utilization in numerical models, for instance. The process of identification requires experimental data obtained from mechanical testing. Most of the previous biomechanical studies on mouse carotid arteries have been conducted using global or average data such as pressure-diameter and/or force-length measurements [8,9]. In addition, the identification of layer-specific parameters of the Holzapfel model has been performed, at this time, only from dissected layers on large arteries [10]. The data which are used in this study are collected with a 3D-DIC stereo-microscopy system on a mouse carotid artery, which is, to our knowledge, unique at this time. See [11] for a description of this previous experimental work.

From experimental data, the identification of constitutive models relies, most of the time, on inverse approaches because establishing response curves from the model may involve complex non-linear relations between the parameters. Classical inverse approaches are based on updating methods [5, 7, 10, 12] using optimization algorithms such as the LevenbergMarquardt algorithm to find the best-fit parameters in a least-square sense with respect to a given cost function. In these approaches, previous authors have most often used analytical developments to derive their modeled data from the given constitutive equations, which presupposed multiple assumptions. Among these, note the widely-accepted assumption of axisymmetry and that of a single homogeneous layer. The latter may be relevant when experimental data is global or averaged over the arterial wall, though two separate layers would be closer to reality and would emphasize the distinction between the layer properties. In addition, for the study of mouse carotid arteries, Gleason [12] performed analytical developments within the frame of thin-tube elasticity theory. This may be a strong assumption in cases of thick arteries like mouse carotid arteries where the ratio of thickness to inner radius was reported to be about $0.6[8,12]$.

Finite element (FE) simulations have rarely been used to recover those modeled data and perform inverse identification. Yet, this kind of approach allows using experimental tests capable of providing richer or otherwise unavailable data and modeling complex problems. Regarding non-linear anisotropic vascular properties, the study of Ning [5] was focused on stress and strain distributions within the arterial wall and how they are influenced by axial pre-stretch. Using the same data as the present study, they identified the parameters of the constitutive model of Bischoff [4], thereby not considering heterogeneity between media and adventitia, which would likely affect these distributions. From our point of view, the advantage of using finite element based identification approaches is to model complex mechanical tests and/or complex structures (see, for instance, [13]), like a thick multi-layer artery presented in this paper.

The question of whether an identification method is relevant with respect to the problem to be treated is seldom addressed. Introducing multiple assumptions and parameters may lead to improper identification or multiple solutions. The objective of the present paper is to address the feasibility of the simultaneous inverse identification of mean fiber angles in both medial and adventitial layers using DIC surface strain measurements.

\section{Methods}

\subsection{Experimental considerations}

The experimental data referenced to in this study were described in deep details in [12] where three-dimensional digital image correlation (3D-DIC) is used to obtain full-field surface strain measurements on mouse carotid arteries at the microscale during an inflation/extension test (Fig. 1). The mechanical test performed here allows both pressurization loading and 
extension loading at the same time (see the schematic principle of the setup in Fig. 1). This test is relevant as it provides biaxial loading conditions close to physiological conditions.

To briefly describe the experimental setup, both ends of a freshly-dissected carotid artery are cannulated with Luer stubs. For image processing and local deformation measurements, a high contrast speckle pattern is incorporated into the vessel structure thanks to ethidium bromide nuclear staining. The experiments are performed with one end of the artery attached to the pressure controller and pressure source, while the other capped end is free in the axial direction, thereby allowing axial translation. The artery is pressurized from 5 to $150 \mathrm{mmHg}$ in steps of $9 \mathrm{mmHg}$ with a flow rate of $0.2 \mathrm{ml} / \mathrm{min}$ and an average pressurization rate of $1.8 \mathrm{mmHg} / \mathrm{s}$. After each pressurization step, synchronized images are acquired from two cameras and analyzed using existing commercial software, VIC-3D.

The region of interest being small (about 200 by $140 \mu \mathrm{m}^{2}$ ), due to the depth of field of the system, and displaying very little heterogeneity, only the strains averaged over this region are considered in the analysis. Note that usual 2DDIC is not suitable for this problem due to the non planar nature of the specimen and possible out-of-plane deformation. More details about the experimental setup and procedure can be found in (Sutton et. al. 2008).

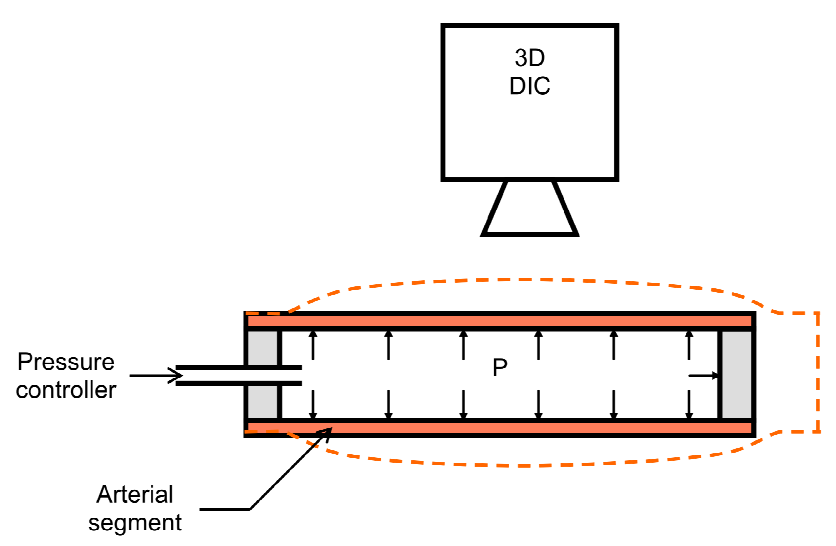

Figure 1. Schematic of the inflation/extension test of the mouse carotid artery showing one fixed end (on the left) linked to the pressure controller and one end (on the right) free to translate axially. Dashed lines represent the schematic shape of the deformed segment.

\subsection{Numerical and constitutive model}

The development of the FE model of the inflation/extension test in Abaqus ${ }^{\circledR}$ is based on the experimental considerations and measurements described in Sutton et al. (2008). The geometry of the artery is assumed to be perfectly cylindrical with one end of the artery being capped. Due to axial symmetry, only one quarter of the geometry is meshed with 4280 8-node brick elements resulting in 22070 degrees of freedom. The element type chosen here, called C3D8RH in Abaqus ${ }^{\circledR}$ (hybrid formulation with constant pressure), is recommended for nearly incompressible constitutive models.

The open end of the cylinder is blocked in the axial direction whereas symmetry boundary conditions are applied on the surfaces of the quarter cylinder. Pressure is applied onto the inner surface of the artery, with values ranging up to $140 \mathrm{mmHg}$.

The constitutive model used in this study is implemented in Abaqus ${ }^{\circledR}$ and based on the developments of Holzapfel [6]. This hyperelastic incompressible model was developed to describe the passive mechanical response of arterial tissues at finite strains. The material considered is a collagen-fiber-reinforced material with two fiber directions being symmetrically arranged with respect to the axis of the artery. This theoretical basis provides a strong physical meaning to the constitutive parameters involved in the model. The simplest form of its isochoric strain energy function consists of two terms (note that incompressibility of the tissue is a well-known characteristic). The first term represents the isotropic response of the medium, related to the ground substance and elastin content, and the other two terms represent the response of the collagenous fiber network, each fiber direction having its own contribution:

$\psi=\frac{C}{2}\left(I_{1}-3\right)+\frac{k_{1}}{2 k_{2}}\left[\exp \left(k_{2}\left(I_{4}-1\right)^{2}\right)-1\right]+\frac{k_{1}}{2 k_{2}}\left[\exp \left(k_{2}\left(I_{6}-1\right)^{2}\right)-1\right]$

where $C$ is the parameter of the isotropic neo-Hookean term, $k_{1}$ and $k_{2}$ are the parameters for the exponential response of the collagen fiber networks. The structural anisotropy induced by the fiber network arises from both $I_{4}$ and $I_{6}$. These terms are pseudo-invariants of the right Cauchy Green tensor $\underline{\underline{\mathrm{C}}}$ and the fiber directions $\underline{\mathbf{f}}_{1}$ and $\underline{\mathbf{f}}_{2}$. Therefore they are driven by $\beta$, the mean fiber angle, in the medium, with respect to the circumferential direction. $I_{4}$ and $I_{6}$ give the squares of stretch for the two fiber families. 
Due to histological differences between media and adventitia, two separate strain energy functions are assigned to each of these mechanically-relevant layers of an artery, the contribution of the intima being commonly considered negligible. Thus, two sets of parameters are to be identified for each layer, yielding eight material parameters. In this study, two variants of this model are considered: a simplified five parameter model and a seven parameter model. The simplified five parameter model includes the assumption that media and adventitia have identical exponential parameters. In addition, it is assumed that the value of $C$ is the same in both layers. Hence, it features the following five parameters: $C$ for the Neo-Hookean isotropic term (elastin and ground substance), two parameters $k_{l}$ and $k_{2}$ for the exponential response of collagen fibers, and two parameters $\beta^{\text {media }}$ and $\beta^{\text {adventitia }}$ for the fiber angles in media and adventitia.

However, a full two-layer Holzapfel-type model considers that the exponential terms used in medial and adventitial layers are different [6], necessitating two additional parameters. Therefore, the seven parameter model releases the constraint on exponential parameters, making them different in each arterial layer.

\subsection{Identification procedure}

Given a set of experimental pressure and surface strain measurements, the principle of the present identification method is to minimize the following cost function:

$$
J(\vec{\chi})=\frac{1}{2}\left[\sum_{i}\left(E_{11}^{\mathrm{sim}}\left(p_{i}\right)-E_{11}^{\mathrm{exp}}\left(p_{i}\right)\right)^{2}+\left(E_{22}^{\mathrm{sim}}\left(p_{i}\right)-E_{22}^{\exp }\left(p_{i}\right)\right)^{2}\right]
$$

where $\vec{\chi}$ is the vector of parameters to be identified (ie. the constitutive parameters), $p_{i}$ is the pressure applied during the inflation test, with index $i$ ranging over the available experimental data points., $E_{11}$ and $E_{22}$ are Green Lagrange circumferential and axial strain components on the surface of the artery, superscripts 'sim' and 'exp' standing respectively for the simulated and experimental data. In this study, synthetic data generated by a FE calculation are also used in order to avoid noise issues when identifying the seven parameter model.

This cost function is minimized using an in-house Levenberg-Marquardt algorithm with bounds handling.

To asses the robustness of the identification method, multiple identification runs with random starting points are performed in order to compare the obtained results. However in the case of the seven parameter model, since noise in data is a major source of identification errors, especially when identifying a lot of parameters, we choose to use noise-free data which are obtained by finite element simulation with an arbitrary set of parameters. Here the set of parameters obtained with the first identification run is used.

\section{Results}

Using the five parameter model, convergence of the optimization algorithm is obtained after 46 iterations. We report in Table 1 the results of this first identification run. The pressure/strain curves are shown in Fig. 2. To further test the identification method, a second set of experimental data obtained by performing a second identical test with the same arterial segment is also used, the aim being to compare the results. We also report in Table 1 the results of this identification run (curves are not shown here). Note that they are very close to those obtained with the first set of data. In the following developments, only the first set of data is used.

In addition to these results, the robustness of the identification method is assessed with the method mentioned in section 2.3 . The range of spanned starting points is chosen according to values found in the literature for this type of artery [12]. The range of the obtained results is reported in Table 1 . Note that $C$ shows quite a large standard deviation because these multiple runs showed that there exist two close minima in the space of parameters, the influence on the response being negligible. 
Regarding the seven parameter model, synthetic noise-free data are generated using the first parameter set identified with the algorithm, referred to as the "true" set. Again, to evaluate the reliability of identifying this constitutive model, we try to identify its seven constitutive parameters using multiple identification random starting points. The results obtained through these tests are presented in Table 1. They raise several noteworthy comments.

The first comment to be made is that all of the runs lead to a very low value of the cost function $J$, which means that the algorithm is practically always able to find a solution. However, only $20 \%$ runs lead to the true set of parameters and the dispersion among the other results is very large.

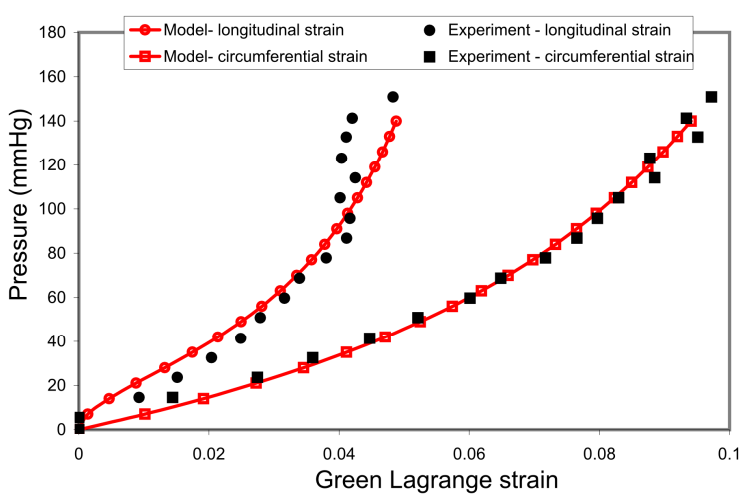

Figure 2. Pressure/strain curves obtained with the five parameter constitutive model.

Table 1. Results of parameter identification for each model and each type of procedure (single run or multiple random starting points run).

\begin{tabular}{|c|c|c|c|c|c|c|c|}
\hline- & $\mathrm{C}(\mathrm{kPa})$ & $\begin{array}{l}\mathrm{k}_{1}{ }^{\text {media }} \\
(\mathrm{kPa})\end{array}$ & $\begin{array}{c}\mathrm{k}_{1}{ }^{\text {adventitia }} \\
(\mathrm{kPa})\end{array}$ & $\begin{array}{l}\mathrm{k}_{2}{ }^{\text {media }} \\
(\mathrm{kPa})\end{array}$ & $\begin{array}{c}\mathrm{k}_{2}^{\text {adventitia }} \\
(\mathrm{kPa})\end{array}$ & $\beta^{\text {media }}\left({ }^{\circ}\right)$ & $\beta^{\text {adventitia }}\left({ }^{\circ}\right)$ \\
\hline Parameter bounds & {$[0.3 ; 50]$} & {$[0.5 ; 100]$} & {$[0.5 ; 100]$} & {$[0.1 ; 100]$} & {$[0.1 ; 100]$} & {$[5 ; 60]$} & {$[5 ; 60]$} \\
\hline- & \multicolumn{7}{|c|}{ Five parameter identification } \\
\hline Results with the first data set & 0.5 & \multicolumn{2}{|c|}{33} & \multicolumn{2}{|c|}{12.8} & 46.4 & 27.2 \\
\hline Results with the second data set & 0.86 & \multicolumn{2}{|c|}{31.3} & \multicolumn{2}{|c|}{14} & 43.3 & 27.3 \\
\hline $\begin{array}{l}\text { Results with random starting points } \\
\text { (mean } \pm \mathrm{SD}) . J \approx 1.10^{-4}\end{array}$ & $0.45 \pm 0.37$ & \multicolumn{2}{|c|}{$30.5 \pm 1.07$} & \multicolumn{2}{|c|}{$15.4 \pm 0.51$} & $46.7 \pm 0.2$ & $26.8 \pm 0.17$ \\
\hline- & \multicolumn{7}{|c|}{ Seven parameter identification } \\
\hline True solution & 8.8 & 13.9 & 11.8 & 21.6 & 0.242 & 41.9 & 5.14 \\
\hline $\begin{array}{l}\text { Results with random starting points } \\
\text { (mean } \pm \mathrm{SD}) . J<1.10^{-5}\end{array}$ & $8.8 \pm 3$ & $12.8 \pm 7.9$ & $19.6 \pm 10$ & $29 \pm 21.7$ & $18.7 \pm 16$ & $41.3 \pm 18$ & $13.3 \pm 13$ \\
\hline- & \multicolumn{7}{|c|}{ Seven parameter identification with enriched cost function } \\
\hline $\begin{array}{l}\text { Solution \#1 } \\
\text { Occurrence } 85.7 \% \text { and } J<1.10^{-8}\end{array}$ & 8.8 & 13.7 & 12.3 & 21.8 & 5.4 & 5.4 & 8 \\
\hline $\begin{array}{l}\text { Solution \#2 } \\
\text { Occurrence } 14.3 \% \text { and } J<1.10^{-5}\end{array}$ & 8.8 & 8.11 & 14.2 & 0.1 & 64.7 & 5 & 38.9 \\
\hline
\end{tabular}

\section{Discussion / conclusion}

\subsection{Five-parameter two-layer model}

In this study, firstly, two separate thick layers are considered, with the further assumption that the material parameters in the exponential terms related to the response of fiber bundles are identical in each layer. This assumption seems to be reasonable as long as only the passive mechanical behavior is considered. The reason for this is that the passive mechanical response is mainly driven by elastin and collagen fibers. The response of elastin, as well as that of the ground substance, is included in the neo-Hookean term of the strain energy function. On the other hand, the response of collagen fibers is included in the exponential terms. Whether they are in media or adventitia, it is assumed in this model that collagen fibers of the arterial wall have the same behavior. 
This parameterization of the constitutive model has been shown to be correctly identified. With any initial values, the method provides a single set of parameters leading to a good agreement with the axial and circumferential experimental data, which proves that the model is well parameterized with respect to the available data and that the method is robust. These results confirm that identifying layer-specific fiber angles, based on the constitutive model of Holzapfel, is possible for such arteries using data from only one experimental test of inflation with free axial movement.

In our results, the mean fiber angle in the media is close to $45^{\circ}$ while fibers are, in average, more circumferentially oriented in the adventitia. This means that the adventitia is found to be circumferentially stiffer than the media. Though it cannot be generalized based on a single example, this result brings some knowledge about the mechanical behavior of these arteries at the layer-scale. The relationship with the actual microstructure remains, however, a pending question [14]. From a global point of view the overall anisotropy of the artery, with the circumferential direction being stiffer, is typical for arteries in general, and is in qualitative agreement with previous studies [11, 12, 14].

\subsection{Seven-parameter model}

In contrast with the five parameter model, the full two-layer Holzapfel-type model has failed in providing a unique solution to the problem of identification. Our results (see Table 1) show the typical trend of an over-parameterized problem. They confirm that identifying such a model on these experimental data is very easy because the algorithm is always able to capture the experimental data with a close fit. Unfortunately, robustness is very poor because seven families of different possible solutions are obtained. Yet, this issue is not unexpected because the problem of inflating a tube made up of two fiberreinforced layers potentially presents two solutions: one solution with a large fiber angle for the inner layer and small angle for the outer layer, and vice versa. Both solutions are possible as long as their constitutive properties can be different to compensate 3D through-thickness effects. Interestingly, our results clearly show these two alternatives, with a noticeable preference for large media angle and low adventitia angle ( $80 \%$ solutions). Note, also, that the mechanism of compensation is clearly illustrated by the ratio of $k_{l}$ parameters between media and adventitia, which is inversed in these two situations.

To correctly identify the parameters for this model, more abundant and/or relevant experimental data are required. In order to confirm this hypothesis, additional synthetic data are generated by simulating a simple axial tension test on the arterial segment, without any pressurization, recording axial force versus axial stretch. Accordingly, the cost function (see Eq. 2) is enriched with these data:

$$
J(\vec{\chi})=\frac{1}{2}\left[\sum_{i}\left(E_{11}^{\mathrm{sim}}\left(p_{i}\right)-E_{11}^{\mathrm{exp}}\left(p_{i}\right)\right)^{2}+\left(E_{22}^{\mathrm{sim}}\left(p_{i}\right)-E_{22}^{\exp }\left(p_{i}\right)\right)^{2}+\sum_{i}\left(F^{\mathrm{sim}}\left(\lambda_{i}\right)-F^{\exp }\left(\lambda_{i}\right)\right)^{2}\right]
$$

where $\lambda_{i}$ is the axial stretch applied during the tension test, with index $i$ ranging over the available experimental (synthetic) data points, $F^{\text {sim }}$ and $F^{\text {exp }}$ are simulated and experimental (synthetic) axial force. Values are scaled so that both sum terms in $J$ are of the same order of magnitude.

Using this updated cost function, the same multiple identification runs are performed again. Instead of obtaining seven different families of solutions, the algorithm provides only two (see Table 1). Among these two solutions, the first one, obtained only $14.3 \%$ times, reaches two boundaries of the allowed range for the parameters. This solution must obviously be rejected, as the procedure would have diverged or found inconsistent values. The other solution is considered as the true solution.

These results show that additional relevant data may easily make the identification procedure robust, discriminating thus the true solution. In this case, a simple tension test added to the inflation/extension test is sufficient.

Circumventing the problem of non-uniqueness emphasized above would also be made possible by using different types of additional data, or other strategies. For instance, separate mechanical tests of medial and adventitial layers can be used as was done in [10], or inversion tests in which the arterial segment is turned inside out to reverse the spatial locations of the media and adventitia and then redistribute through-thickness stresses in a different manner (theoretically studied in [2]). Another possible way to access useful experimental information would be to acquire through-thickness data, thanks to the use of optical coherence tomography or confocal microscopy, for instance. Such layer-specific data would help discriminate the true solution. Otherwise, in order to reduce the number of dependent parameters to be identified and help make the solution unique, additional information regarding the fiber angle distributions within each layer (from histology for instance) would be helpful. A part of the work in progress at this time is related to these last two aspects. 


\section{References}

[1] Fung YC, Biorheology of soft tissues, Biorheology, 10, 139-55, 1973.

[2] Humphrey JD, Cardiovascular solid mechanics: cells, tissues, organs, New York: Springer-Verlag, 2002.

[3] Vito RP, Dixon SA, Blood vessel constitutive models, Annu Rev Biomed Eng, 5, 413-39, 2003.

[4] Bischoff JE, Arruda EA, Grosh K, A Microstructurally Based Orthotropic Hyperelastic Constitutive Law, J Appl Mech, 69(5), 570-579, 2002.

[5] Ning J, Xu S, Wang Y, Lessner SM, Sutton, MA, Anderson K, Bischoff JE, Deformation measurements and material property estimation of mouse carotid artery using a microstructure-based constitutive model, J Biomech Eng, 132, DOI:10,1115/1,4002700, 2010.

[6] Holzapfel GA, Gasser TC, Ogden RW, A New Constitutive Framework for Arterial Wall Mechanics and a Comparative Study of Material Models, J Elasticity, 61, 1-48, 2000.

[7] Masson I, Boutouyrie P, Laurent S, Humphrey JD, Zidi M, Characterization of arterial wall mechanical behavior and stresses from human clinical data, J Biomech, 41, 2618-2627, 2008.

[8] Dye WW, Gleason RL, Wilson E, Humphrey JD, Altered biomechanical properties of carotid arteries in two mouse models of muscular dystrophy, J Appl Physiol, 103, 664-672, 2007.

[9] Guo X, Oldham MJ, Kleinman MT, Phalen RF, Kassab GS, Effect of cigarette smoking on nitric oxide, structural, and mechanical properties of mouse arteries, Am J Physiol Heart Circ Physiol 291, H2354-H2361, 2006.

[10] Holzapfel GA, Sommer G, Gasser TC, Regitnig P, Determination of the layer-specific mechanical properties of human coronary arteries with non-atherosclerotic intimal thickening, and related constitutive modelling, Am J Physiol Heart Circ Physiol, 289, 2048-2058, 2005.

[11] Sutton MA, Ke X, Lessner SM, Goldbach M, Yost M, Zhao F, Schreier HW, Strain field measurements on mouse carotid arteries using microscopic three-dimensional digital image correlation, J Biomed Mater Res Part A, 84, 178-190, 2008 .

[12] Gleason RL, Dye WW, Wilson E, Humphrey JD, Quantification of the mechanical behavior of carotid arteries from wild-type, dystrophin-deficient, and sarcoglycan- $\delta$ knockout mice, J Biomech, 41, 3213-3218, 2008.

[13] Avril S, Badel P, Duprey A, Anisotropic and hyperelastic identification of in vitro human arteries from full-field measurements, J Biomech, 43 (15), 2978-2985, 2010.

[14] Haskett D, Johnson G, Zhou A, Utzinger U, Vande Geest J, Microstructural and biomechanical alterations of the human aorta as a function of age and location, Biomech Model Mechanobiol, 9, 725-736, 2010. 